\title{
Criteria for Enrolling Dementia Patients in Hospice
}

\author{
Daniel J Luchins, MD, "Patricia Hanrahan, PhD, and Kathleen Murphy, $M D^{\dagger}$
}

OBJECTIVE: Because survival time varies greatly, it is difficult for dementia patients to meet a key criterion for eligibility for the Medicare hospice benefit: a 6-month survival time. We have developed criteria for the Medicare hospice benefit that include the characteristics of advanced dementia and related medical complications. The purpose of the study was to determine survival time among dementia patients who met these criteria. Additionally, because the National Hospice Organization (NHO) developed its own guidelines while the study was in progress, we retrospectively examined the application of these guidelines to our sample.

DESIGN: Two cohorts of hospice patients were studied longitudinally, each for 2 years.

SETTING: Nine Midwestern hospice programs.

PARTICIPANTS: Forty-seven patients were enrolled in home hospice and institutional hospice settings.

MEASUREMENTS: Survival time consisted of the number of days between enrollment in the hospice program and death or the end of the study. Other measures included Activities of Daily Living, ratings of Appetite, Nourishment, and Mobility, Functional Assessment Staging (FAST), a Medical Complications Checklist, and a care plan concerning the use of medications for acute illness.

RESULTS: Our hospice enrollment criteria predicted a median survival time of 4 months and a mean survival time of 6.9 months; $38 \%$ of patients survived for more than 6 months. FAST scores and Mobility ratings were significantly related to survival time. However, $41 \%$ could not be scored on the FAST as their disease progression was not ordinal. Among patients who could be scored on the FAST and who had reached Stage Seven C, their mean survival time was 3.2 months compared with 18 months among those who could be scored and had not reached this stage and 8.6 months among patients whose disease progression was not ordinal,

From "The University of Chicago and the Illinois Department of Mental Health and Developmental Disabilities, and tHospice of Michigan.

This research was supported by The University of Chicago Hospitals' Home Care Research Grants, the Alzheimer's Association/Mr. and Mrs. Neil Bluhm Pilot Research Grant, the Alzheimer's Disease Research Committee of the Illinois Department of Public Health, and Henry Ford Health Systems.

Preliminary findings were presented at the annual conference of the American Psychiatric Association in Miami, Florida, 1995; the Hastings Center Conference on Dying with Dementia. Briarcliff Manor, New York, 1996; the annual conference of the National Hospice Organization, Chicago, Illinois, 1996; and the annual Institute on Psychiatric Services, Chicago, Illinois, 1996.

Address correspondence and reprint requests to Daniel J. Luchins, MD, The University of Chicago, Dept. of Psychiatry, MC3077, 5841 S. Maryland Ave., Chicago, IL 60637.
$P<.001$. When the palliative care plans were examined, less aggressive care plans resulted in shorter survival times, $P<$ .01 .

CONCLUSION: Our hospice enrollment criteria identified a group with a median survival time of 4 months and a mean survival time of 6.9 months. Using $\mathrm{NHO}$ criteria relying on the FAST allows the identification of a subgroup with very high mortality and a short time until death. Although the FAST can identify a subgroup of appropriate candidates for hospice, sole reliance on this measure might decrease access to hospice care for many dementia patients. J Am Geriatr Soc 45:1054-1059, 1997.

$\mathrm{E}_{\mathrm{w}}^{\mathrm{n}}$ d-stage dementia is a significant clinical problem for which alternative modes of care are needed. Many patients with end stage dementia are ideal candidates for hospice because their prognosis is terminal and aggressive medical treatment is rarely preferred. ${ }^{1-2}$ Palliative care is intended to relieve symptoms, to control pain, and, thereby, to ease death rather than to prolong life. These goals apply to endstage dementia patients, who often die of medical complications of the disease such as aspiration pneumonia. Reports of pilot hospice programs for dementia patients suggest that this approach is feasible ${ }^{3-5}$ as well as ethical. ${ }^{16-8}$ In one survey, $90 \%$ of family and professional caregivers of dementia patients viewed hospice care as appropriate for the end stages of the disease. ${ }^{2}$ Yet only $13 \%$ were aware of hospices that served dementia patients compared with $87 \%$ who were aware of hospice services for cancer patients. This suggests unmet need.

\section{For editorial comment, see p. 1147}

Lack of utilization of hospice care in advanced dementia was further documented in our national survey of 1184 hospice programs. ${ }^{9}$ Fewer than $1 \%$ of hospice patients had a primary diagnosis of dementia. A study that examined Medicare beneficiaries in hospice had similar findings; $1.5 \%$ of hospice patients had dementia as a primary diagnosis. ${ }^{10}$ Among hospice staff from our national survey, $80 \%$ viewed difficulties in predicting the survival time of dementia patients as a major problem in their efforts to serve these patients. Eligibility for the Medicare hospice benefit requires a prognosis of death within 6 months. However, survival time in Alzheimer's disease and vascular dementia is highly variable and can range from 2 to 10 years. ${ }^{11}$ Recent evidence has confirmed that dementia patients in hospice have a significantly longer survival time than those with other diagnoses, 
with $35 \%$ surviving longer than 6 months. ${ }^{10}$ Yet relatively little research has been conducted on the development of empirically based standards for enrolling patients with endstage dementia in hospice, aside from the pioneering studies of Volicer and his colleagues in an institutional hospice.,12

In a study of a home-based hospice, we have developed enrollment criteria that identified a group of dementia patients whose median survival time was 5 months, with a mean of 7 months (Table 1). ${ }^{5}$ The main purpose of this research was to expand the study to institutional hospice settings as well as to replicate our findings with home hospice patients.

A second purpose concerns the analysis of the National Hospice Organization (NHO) guidelines. Since these studies began, NHO guidelines have been developed to assist primary care physicians to identify "patients with nononcological disease who may be appropriate for admission to a hospice program. . . under the Medicare/Medicaid Hospice Benefit." ${ }^{13}$ Medical guidelines for assessing prognosis were developed for other terminal illnesses in addition to dementia, for example, heart disease and pulmonary disease. It is anticipated that a revised version will include more guidelines for other end-stage conditions. The guidelines are designed not to function as strict admission criteria but to assist physicians in making clinical judgments. With regard to dementia, the NHO guidelines parallel our own but with the addition of a more detailed description of medical complications and the suggestion that Stage Seven $\mathrm{C}$ of Functional Assessment Staging may be an appropriate cutoff point for enrollment (Table 2). ${ }^{13,14}$ Using our experience with the patients in our study, we have examined the implications of these refinements.

\section{Table 1. Hospice Enrollment Criteria for End-Stage Dementia Patients ${ }^{5}$}

Signs of very severe cognitive decline

May have the following (if known)

Low mental status scores (MSQ or MMSE less than or equal to 1)

All of the following impairments due to severe cognitive decline

Incontinent

Needs assistance with eating

Needs assistance with walking

Needs assistance with bathing and grooming

Very limited speech or cannot communicate meaningfully

Unable to engage in purposeful activities

Serious complications: Patient should have a current or recent history of one or more of the following impairments

Difficulty swallowing food

Aspiration pneumonia

Dehydration

Malnutrition

Severe urinary tract infection

Decubitus ulcers

Septicemia

Other serious complications
Table 2. NHO Medical Guidelines for Determining Prognosis in Dementia ${ }^{13}$

Functional assessment staging

Patients who have reached the level of severity of dementia described here may have a prognosis of up to 2 years

At or beyond Stage Seven of the Functional Assessment Staging Scale

All of the following characteristics

Unable to dress without assistance

Unable to bathe properly

Urinary and fecal incontinence

Unable to speak or communicate meaningfully

Unable to ambulate without assistance

Presence of medical complications

Comorbid conditions of sufficient severity to warrant medical treatment, documented within the past year

Comorbid conditions associated with dementia

Aspiration pneumonia

Pyelonephritis or other upper urinary tract infection

Septicemia

Decubitus ulcers

Difficulty swallowing food or refusal to eat, sufficiently severe that patient cannot maintain sufficient fluid and calorie intake to sustain life*

Stage Seven C of the FAST may be an appropriate enrollment cutoff (Appendix 1). At this stage, patients are usually

Either mute or limited to a single intelligible word in the course of the day,

Dependent in all Activities of Daily Living, including mobility.

* Patients who are receiving tube feeding must have documented Impaired Nutritional Status

\section{METHODS}

\section{Patients}

Enrollment criteria developed for the study consisted of advanced dementia and a history of medical complications during the year before enrollment (Table 1). Patients were eligible for hospice if they were severely demented, with very low mental status, needed assistance in most Activities of Daily Living, and were no longer able to communicate beyond an occasional word or two. Patients were also required to have a recent history of complications typical of end-stage dementia, for example, aspiration pneumonia, which causes swallowing problems. Patients who fit the criteria were enrolled in the following two cohorts: (1) Meridian Hospice between 11/1/90 and 10/31/92 $(n=11)$ and (2) nine Midwestern hospices between 4/1/93 and 3/31/95 ( $\mathrm{n}=36$ ). Enrollment stopped 6 months before the end of each 2-year study period in order to allow for a 6-month follow-up period. No information is available about those patients who did not meet the criteria for admission to hospice although anecdotal evidence from hospice staff suggests that there were very few such patients.

\section{Settings}

Settings included the following nine Midwestern hospices: Meridian Hospice, Henry Ford Hospice, LaGrange 
Memorial Hospice, Hospice of the North Shore, Horizon Hospice, Evangelical Health Service Hospice, West Towns VNS Hospice, VNA Hospice of Chicago, and the Hospice of Northeastern Illinois. Care was provided both at home and in nursing homes

\section{Measures}

After training in the use of the assessment instruments, hospice nurses collected patient information for the research staff. Because the hospice staffs were not accustomed to assessing the severity of dementia, a range of measures was used both to assist them in making this assessment and to establish baseline levels of disability. The following measures were included.

\section{Cognitive Impairment}

The Mental Status Quotient ${ }^{15}$ was used to establish cognitive impairment.

\section{Functional Impairment Levels}

Activities of Daily Living were rated on a 3-point scale (independent, needs assistance, totally dependent) from the Older Adults Resources and Services Instrument (OARS). ${ }^{16-17}$ For example, with regard to eating, staff were asked to rate whether the patient could eat: (1) Without help (can feed self completely), (2) With some help (needs help cutting, etc.), or was (3) Completely unable to feed self.

\section{Medical Complications Checklist}

The Medical Complications Checklist (MCC) consisted of a list of common complications of end-stage dementia and when the problem occurred (current vs within the past year). Complications included swallowing problems, aspiration pneumonia, malnutrition, dehydration, decubitus ulcers, urinary tract infections, septicemia, seizures, fractures or broken bones, and deep vein thrombosis. The checklist was based on typical complications in advanced dementia according to physicians who responded to our previous survey. ${ }^{2}$

\section{Survival Time}

Survival Time consisted of the number of days between enrollment in the hospice program and the patient's death, or if patients were not deceased, the number of days from enrollment until 10/31/92 for the first cohort and until $3 / 31 / 95$ for the second cohort.

\section{Additional Measures}

Additional Measures were used with the second cohort in order to further characterize factors influencing survival time:

\section{Performance Ratings}

These were drawn from a study of survival time among cancer patients in hospice: Appetite: $1=$ normal to $5=$ refuses fluid; Nourishment: $1=$ heavy to $5=$ extreme cachexia; and Mobility: $1=$ normal to $5=$ unable to turn in bed. ${ }^{18}$

\section{Level of Deterioration}

In the Functional Assessment Staging (FAST), the seven stages of previously dementia described in the Global Deterioration Scale (GDS) are further elaborated, and an ordinal progression is assumed (Appendix ). ${ }^{14,19}$ For example, Stage
Seven is the most severe stage of dementia in the GDS. In the FAST, Stage Seven has been further subdivided to include six additional levels of disease progression in which the following deficits are assumed to occur in sequential order. At Stage Seven A, speech is limited to about six words in the course of a day. Next, at Stage Seven B, intelligible vocabulary is limited to a single word in the course of the day. Subsequently, at Stage Seven C, ambulatory ability becomes impaired, and physical assistance is needed to walk. These impairments are followed by the loss of the ability to sit up (7D), to smile (7E), and to hold the head up (7F).

\section{Palliative Care Plan}

Hospice staff were asked to characterize the palliative care plan with regard to whether or not medications could be used for acute illnesses.

\section{Data Analysis}

Descriptive statistics were used to characterize demographics, diagnosis, and survival time. Initially, univariate analysis was used to identify variables related to survival time (i.e., the correlation between performance ratings of mobility and survival time). Significant univariate relationships were then examined using multiple regression. With regard to NHO guidelines, a one-way ANOVA was used to compare survival times among those whose impairment was characteristic of Stage Seven C of the FAST, those who had not yet reached Stage Seven $C$, and those who could not be scored (see results for further discussion of this issue). Additionally, the combined effect of the patient's care plan and status on the FAST was examined using a two-factor ANOVA.

\section{RESULTS}

\section{Subjects}

orty-seven patients were enrolled in hospice care. The majority were women $(70 \%)$. Their average age was 84 $(\mathrm{SD}=9.2)$. The majority $(66 \%)$ were white $(66 \%)$, and the remainder were black. Most had Alzheimer's disease (71\%). The remainder had vascular disease $(9 \%)$, a mixed dementia, usually vascular disease and Alzheimer's disease $(6 \%)$, Parkinson's disease $(6 \%)$, or an unspecified dementia $(8 \%)$. As per the enrollment criteria, all clients were severely impaired in their capacity to perform Activities of Daily Living (ADL) and had medical complications related to their dementia. Eighty-six percent had more than one medical complication. The majority had swallowing problems $(72 \%)$. Decubitus ulcers also occurred frequently $(70 \%)$. Aspiration pneumonia was common $(55 \%)$, as were dehydration $(57 \%)$, malnutrition $(50 \%)$, and urinary tract infections $(37 \%)$. The patients' cognitive impairments were so severe that most were unable to participate in tests of mental status. The severity of the dementia corresponded to Stage Seven on the Global Deterioration Scale for all but one of the patients, who was in Stage Six E of the FAST. ${ }^{14}$

\section{Median Survival Time}

Among the 47 patients, median survival time was 4 months, with an average of 6.9 months ( $S D=7.3$ months); $18(38 \%)$ survived longer than 6 months. The 37 deceased patients from both cohorts had a mean survival time of 4.5 months ( $\mathrm{SD}=6.5$ months). The average survival time for the 10 patients $(21 \%)$ who were still alive was 15.7 months 
( $\mathrm{SD}=6.5$ months). Patient characteristics such as demographics and diagnosis were not significantly related to their survival time. There were no significant differences in survival time between patients receiving hospice at home $(n=22)$ and those receiving institutional hospice $(n=25)$.

\section{Predictors of Survival Time}

Predictors of survival time were examined only for patients in the second cohort and are shown below.

\section{FAST Scores}

FAST scores were the strongest predictor, $(r=-.66, P<$ $.01, \mathrm{n}=17$ ). However, as discussed in a later section, many patients could not be scored.

\section{Performance Ratings}

Mobility impairments were highly correlated with survival times $(r=-.55, P<.001, \mathrm{n}=32)$, as were ratings of Appetite $(r=-.47, P<.01, \mathrm{n}=29)$. Nourishment was not significant $(r=-.24, P=.19, \mathrm{n}=32)$. Functional impairment, as indicated by a total score on Activities of Daily Living, was also a strong predictor of survival time, $(r=.51$, $P<.01, \mathrm{n}=32$ ).

\section{Medical Complications}

It was not possible to examine the role of specific comorbidities, because most patients had more than one medical complication. However, the more complications the patients had, the shorter their survival time $(r=-41, P<.05, \mathrm{n}=29)$.

\section{Multivariate Analysis}

Multivariate analysis using multipie regression was conducted next to examine the relation between survival time and those predictors that were significant using univariate analysis. FAST scores were excluded from this analysis because only 17 patients could be assessed (as discussed below). Only performance ratings of mobility remained significant in this analysis $(\mathrm{F}=12.05, P<.01)$ and accounted for $28 \%$ of the variance in survival time (Adjusted $R^{2}=28.3, n^{\prime}=29$ to 32).

\section{Problems in Using the FAST}

Because of time constraints, hospice staff were often unable to score the FAST, resulting in missing data for $19 \%$ of the second cohort $(n=7)$. A more important issue was that the assumption of an ordinal pattern of deterioration in the FAST proved to be inaccurate for $12(41 \%)$ of the 29 patients for whom this data was available (see Appendix). As a result, patients were next grouped in one of the following three categories:

\section{Patient Has Not Deteriorated to the Point of Reaching Stage Seven Cor Greater $(n=5)$}

Because the enrollment criteria suggested that patients have impairments in most or all ADLs, relatively few patients were in this group. One of these patients was at Stage Six E at intake, and the others were at Stage Seven A $(n=2)$ or Stage Seven $B(n=2)$. Although their verbal ability varied, the ability of these patients to walk was not impaired. The most striking finding was that all patients who could be scored in an ordinal fashion and who had not reached Stage Seven C were still surviving, with a mean survival time of 18.1 months
( $S D=7.1$ months). All of these patients survived for more than 6 months.

\section{Patient Has Deteriorated to Stage Seven C or Greater $(n=12)$}

The distinguishing characteristic of Stage Seven C is that, in addition to being limited to one word a day, ambulation is impaired. Mean survival time was 3.2 months $(\mathrm{SD}=3.8$ months), and 11 of 12 patients (92\%) were deceased. Two patients $(17 \%)$ survived longer than 6 months.

\section{Patient Did Not Follow an Ordinal Progression ( $n=12)$}

The major difficulty in scoring the FAST in this group was their intelligible vocabulary had not decreased to one word a day. They could not, therefore, be coded as having progressed through Stage 7B, yet they had mobility impairments that would otherwise be characteristic of Stage 7C. Because their verbal impairment had not decreased to Stage Seven B (single word/day), their disease progression did not follow the sequence necessary for their assessment on the FAST. Within this group, the mean survival time was 8.6 months (SD $=7$ months); 11 of 12 patients $(92 \%)$ were deceased but seven $(58 \%)$ survived for more than 6 months.

Survival times among these three groups differed significantly, $P<.001$ (ANOVA, $F=11.52$ ). All pairwise comparisons were also significant (Tukey-B test, $P<.05$ ).

\section{Palliative Care Plan}

When both FAST status and care plans were examined jointly, each had an effect on survival times (Table 3). Patients whose care plans called for the use of medication for acute illness had longer survival times than those without this provision, $P<.01$. There were no significant interactions.

\section{Limitation}

The small sample size limited multivariate analysis to the detection of large effect sizes. However, a large effect could be expected to have clinical significance in decision-making. Further research with a larger sample would be useful in

Table 3. Survival Time: Effect of Palliative Care Plan and Patient Status on the FAST

\begin{tabular}{|c|c|c|c|}
\hline FAST Status* & $N=25$ & $\begin{array}{l}\text { Medications } \\
\text { Used for } \\
\text { Acute Illness } \\
\text { Survival Time }\end{array}$ & $\begin{array}{c}\text { Medications } \\
\text { Not Used } \\
\text { Survival } \\
\text { Time }\end{array}$ \\
\hline $\begin{array}{l}\text { More intact than } \\
\text { Stage } 7 \mathrm{C}\end{array}$ & 4 & $\begin{array}{c}24.2 \text { months } \\
(\mathrm{SD}=.2) \\
\mathrm{n}=2\end{array}$ & $\begin{array}{c}17.5 \text { months } \\
(\mathrm{SD}=2.8) \\
\mathrm{n}=2\end{array}$ \\
\hline $\begin{array}{l}\text { At or past } \\
\text { Stage } 7 \mathrm{C}\end{array}$ & 11 & $\begin{array}{c}4 \text { months } \\
(S D=3.9) \\
n=6\end{array}$ & $\begin{array}{c}2.6 \text { months } \\
(S D=4.3) \\
n=5\end{array}$ \\
\hline Not ordinal & 10 & $\begin{array}{c}14.9 \text { months } \\
(\mathrm{SD}=6.2) \\
n=5\end{array}$ & $\begin{array}{c}5.2 \text { months } \\
(\mathrm{SD}=3) \\
\mathrm{n}=5\end{array}$ \\
\hline
\end{tabular}

ANOVA, $\mathrm{F}=19.95, P<.001$,

*FAST Status, Main Effect, $F=25.6, P<.001$

${ }^{*}$ Use of Medications, Main Effect, $F=9.8, P<.01$ 
examining such issues as the relative importance of specific medical complications.

\section{DISCUSSION}

Our proposed hospice enrollment criteria identified a group of dementia patients with a median survival time of 4 months; $38 \%$ survived past 6 months. These results are similar to the median survival of 2.5 months, with $35 \%$ living past 6 months, reported in a national study of Medicare hospice beneficiaries with a primary diagnosis of dementia. ${ }^{10}$ Although all the patients in our study were severely demented, there were still gradations of disability that our subsequent analysis shows are related to differing survival patterns. Patients who can be scored on the FAST and who have not reached Stage Seven C are unlikely to die within 6 months. These patients do not appear to fit current requirements for eligibility for hospice. In contrast, patients who can be scored on the FAST and who have reached Stage Seven C appear to be good candidates for hospice as most of those enrolled in the study died (11 of 12), with a mean survival time of 3.2 months, and only two patients $(17 \%)$ survived more than 6 months.

It is the patient whose disease progression was not ordinal who poses problems in using the FAST as an enrollment criterion. Problems in the assumption of an ordinal deterioration in dementia have been identified in our previous research using a sample from an Alzheimer's Disease Registry. ${ }^{20}$ The use of Stage Seven $C$ as a cutoff is problematic because the lack of ordinality precluded using this measure for almost half of the patients in the second cohort. The non-ordinal patients had impaired mobility consistent with Stage Seven $C$ but less severely impaired language function (i.e., greater than one word a day).

It is not clear whether the Medicare policy for a 6-month prognosis refers to a median survival time, a mean survival time, or that $75 \%$, or $85 \%$, or $95 \%$ should be deceased at that point. ${ }^{21}$ Obviously, the more stringent the interpretation, the more patients would lose access to hospice services. This issue is of particular importance for those severely demented patients whose disease progression did not follow an ordinal pattern. One possible way of dealing with these patients would be to assume that further deterioration, for instance, in their scant remaining language ability, might occur, and that hospice admission could be delayed until this deterioration took place. They would then be at Stage Seven $\mathrm{C}$, regardless of initial ordinality. Inasmuch as our study did not measure deterioration on the FAST over time, we cannot analyze this possibility directly. Because the FAST was only available at hospice enrollment, we have instead examined how long these patients survived in hospice in order to estimate what proportion would have survived if hospice admission were delayed for varying time periods in order to reevaluate their deterioration. Within 1 month of hospice enrollment one such patient died ( $8 \%$ of this group). By the third month of hospice care, another three patients died $(25 \%)$; one more died in the fourth month for a total of five or $42 \%$ within 4 months of hospice enrollment. If strict adherence to Stage Seven C of the FAST is required before enrollment, then frequent re-evaluation of non-ordinal patients would be necessary or many of these patients would have died without benefit of hospice.

This analysis may underestimate the impact of delay on enrollment because it ignores the effect of treatment on sur- vival. For the non-ordinal group, even though all were enrolled in hospice, there was a 3-fold difference in survival between those who received (14.9 months) and those who did not receive (5.2 months) medications for acute illness. This finding is similar to the life-prolonging effect of treating fever observed by Volicer and his colleagues in those with less severe disability. ${ }^{12}$ This effect of treatment suggests that non-ordinal end stage dementia patients, with impaired mobility and better preserved language, might be suitable for hospice if their palliative care plans were conservative and not suitable if more life-prolonging care was anticipated.

In summary, our findings and this discussion would support the use of the NHO guidelines for enrolling dementia patients. Those individuals who clearly meet the guidelines are appropriate for the Medicare hospice benefit, whereas those who clearly do not meet the guidelines are not appropriate. Thus the NHO guidelines - when applicable - offer a very useful set of criteria for hospice enrollment with eligibility for Medicare reimbursement. The problem we have identified and tried to address is that of individuals who cannot be categorized on the FAST because their progression is not ordinal.

It is possible that with additional iterations, some modification of our criteria and/or procedures for dealing with individuals who do not progress ordinally on the FAST might be shown to define a group of end stage dementia patients who fulfill the requirement of dying in 6 months. However, one could question this effort and consider alternative approaches. When the admission guideline concerning a 6-months survival time was initially adopted for the Medicare hospice benefit, most hospice patients were dying of cancer. The use of this guideline for the new groups of patients that hospices have begun to serve, notably dementia patients and AIDS patients, may not be appropriate. Although hospice programs have expanded greatly since their inception, only about $15 \%$ of terminal patients receive hospice services annually. ${ }^{22}$ Alternatives to the Medicare hospice benefit have been proposed for patients who are not dying of cancer, but at this point hospice care is the only major program organized specifically for the needs of dying patients. ${ }^{23}$ In order to make this important service more accessible, less rigorous admissions criteria have been suggested for AIDS patients, for example, a limited prognosis and a need for skilled nursing. ${ }^{24}$ Similar flexibility may be needed for end-stage dementia patients.

\section{ACKNOWLEDGMENTS}

This research was made possible by generous support from The University of Chicago Hospitals' Home Care Research Grant, the Alzheimer's Association/Mr. and Mrs. Neil Bluhm Pilot Research Grant, Henry Ford Health Systems, and a research award from the Alzheimer's Disease Research Committee of the Illinois Department of Public Health. For their assistance with our study, we are also very grateful for the efforts of the hospice staffs in extending hospice to a new population, dementia patients. The authors also appreciated the thoughtful reviews of the manuscript by Greg Sachs, MD, of The University of Chicago's Center on Aging, Health and Society, Diane Meier, MD, of Mount Sinai Medical Center, and Brad Stuart, MD, of the NHO Medical Guidelines Task Force. 


\section{REFERENCES}

1. Volicer L. Need for hospice approach to treatment of patients with advanced progressive dementia. J Am Geriatr Soc 1986;34:655-658.

2. Luchins DJ, Hanrahan $P$. What is the appropriate level of health care for end-stage dementia patients? J Am Geriatr Soc 1993;41:25-30.

3. Volicer L, Rheaume $\mathrm{Y}$, Brown J et al. Hospice approach to the treatment of patients with advanced dementia of the Alzheimer type. JAMA 1986;256:2210-2213.

4. Brechling BG, Heyworth J, Kuhn D, Peranteau MF. Extending hospice care to end-stage dementia patients and families. Am J Alzheimer Care Rel Disord Res 1989;21-29.

5. Hanrahan P, Luchins DJ. Feasible criteria for enrolling end-stage dementia patients in home hospice care. Hospice J 1995;10:47-54.

6. President's Commission for the Study of Ethical Principles in Medicine and Biomedical and Behavioral Research: Deciding to Forego Life-Sustaining Treatment. Washington, DC: Government Printing Office, 1983.

7. Myers RM, Grodin MA. Decision-making regarding the initiation of tube feedings in the severely demented elderly: A review. J Am Geriatr Soc 1991;39:526-531.

8. Lynn J. Dying and dementia. JAMA 1986;256:2244-2245.

9. Hanrahan P, Luchins DJ. Access to hospice care for end-stage dementia patients: A national survey of hospice programs. J Am Geriatr Soc 1995;43:56-59.

10. Christakis NA, Escarce JJ. Survival of Medicare patients after enrollment in hospice programs. N Engl J Med 1996;335:172-178.

11. Barclay LL, Zemcov A, Blass JP et al. Survival in Alzheimer's disease and vascular dementias. Neurology 1985;35:834-840.

12. Volicer BJ, Hurley A, Fabiszewski KJ et al. Predicting short-term survival for patients with advanced Alzheimer's disease. J Am Geriatr Soc 1993;41:535-540.

13. NHO, Stuart B, Herbst L, Kinzbrunner B et al. Medical guidelines for determining prognosis in selected non-cancer diseases. Hospice J 1996;11:47-63.

14. Reisberg B. Functional Assessment Staging (FAST). Psychopharm Bull 1988;24:653-659.

15. Kahn RL, Goldfarb AI, Pollack M, Peck A. Brief objective measures for the de termination of mental status in the aged. Am J Psychiatry 1960;117:326-328.

16. Katz S, Hedrick S, Henderson N. Studies of illness in the aged: The Index of ADL: A standardized measure of biological and psychosocial function. JAMA 1963;185:914-919.

17. Whanger AD, Myers AC, eds. Mental Health Assessment and Therapeutic Intervention with Older Adults. Rockville, MD: Aspen Publications, 1984.

18. Schonwetter RS, Teasdale TA, Storey $P$ et al. Estimation of survival time in terminal cancer patients: An impedance to admission. Hospice J 1990;6:65-80.

19. Reisberg B, Ferris SH, De Leon MJ, Crook T. The Global Deterioration Scale for assessment of primary degenerative dementia. Am J Psychiatry 1982;139:1136-1139.

20. Eisdorfer C, Cohen D, Paveza GJ et al. An empirical evaluation of the Global Deterioration Scale for staging Alzheimer's disease. Am J Psychiatry 1992;149:190-194.

21. Sachs GA, Ahronheim JC, Rhymes JA et al. Good care of dying patients: The alternative to physician assisted suicide and euthanasia. J Am Geriatr Soc 1995;43:553-562.

22. Mahoney JJ, Miller G. What does hospice offer Alzheimer's patients and families? Hastings Center Conference on Dying with Dementia. Briarcliff Manor, NY: Hastings Center, 1996.

23. Lynn J. Caring at the end of our lives. N Engl J Med 1996;335:201-202.
24. Beresford. L. The challenge of AIDS. California Hospice Report 1989;1:116.

\section{APPENDIX}

\section{FUNCTIONAL ASSESSMENT STAGING (FAST, 14)}

(Check highest consecutive level of disability)

1. No difficulty either subjectively or objectively

2. Complains of forgetting location of objects. Subjective work difficulties

3. Decreased job functioning evident to co-workers. Difficulty in traveling to new locations. Decreased organizational capacity*

4. Decreased ability to perform complex tasks, e.g., planning dinner for guests, handling personal finances (such as forgetting to pay bills), difficulty marketing, etc

5. Requires assistance in choosing proper clothing to wear for the day, season or occasion, e.g., patients may wear the same clothing repeatedly, unless supervised*

6. A) Improperly putting on clothes without assistance or cuing (e.g., may put street clothes on over night clothes, or put shoes on wrong feet, or have difficulty buttoning clothing) occasionally or more frequently over the past weeks*

B) Unable to bathe properly (e.g., difficulty adjusting bath-water temperature) occasionally or more frequently over the past weeks*

C) Inability to handle mechanics of toileting (e.g., forgets to flush the toilet, does not wipe properly or properly dispose of toilet tissue) occasionally or more frequently over the past weeks ${ }^{*}$

D) Urinary incontinence (occasionally or more frequently over the past weeks)*

E) Fecal Incontinence (occasionally or more frequently over the past weeks) ${ }^{\star}$

7. A) Ability to speak limited to approximately a half a dozen intelligible different words or fewer, in the course of an average day or in the course of an intensive interview

B) Speech ability is limited to the use of a single intelligible work in an average day or in the course of an intensive interview (the person may repeat the word over and over)

C) Ambulatory ability is lost (cannot walk without personal assistance)

D) Cannot sit up without assistance (e.g., the individual will fall over if there are not lateral rests (arms) on the chair)

E) Loss of ability to smile

F) Loss of ability to hold up head independently

* Scored primarily on the basis of information obtained from a knowledgeable informant and/or category. 